\title{
On the Existence of Solutions for the Critical Fractional Laplacian Equation in $\mathbb{R}^{N}$
}

\author{
Zifei Shen and Fashun Gao \\ Department of Mathematics, Zhejiang Normal University, Jinhua 321004, China \\ Correspondence should be addressed to Zifei Shen; szf@zjnu.cn \\ Received 5 May 2013; Accepted 10 December 2013; Published 12 January 2014 \\ Academic Editor: Wenming Zou
}

Copyright (c) 2014 Z. Shen and F. Gao. This is an open access article distributed under the Creative Commons Attribution License, which permits unrestricted use, distribution, and reproduction in any medium, provided the original work is properly cited.

\begin{abstract}
We study existence of solutions for the fractional Laplacian equation $(-\Delta)^{s} u+V(x) u=|u|^{2^{*}(s)-2} u+f(x, u)$ in $\mathbb{R}^{N}, u \in H^{s}\left(R^{N}\right)$, with critical exponent $2^{*}(s)=2 N /(N-2 s), N>2 s, s \in(0,1)$, where $V(x) \geq 0$ has a potential well and $f: \mathbb{R}^{N} \times \mathbb{R} \rightarrow \mathbb{R}$ is a lower order perturbation of the critical power $|u|^{2^{*}(s)-2} u$. By employing the variational method, we prove the existence of nontrivial solutions for the equation.
\end{abstract}

\section{Introduction}

In the last 20 years, the classical nonlinear Schrödinger equation has been extensively studied by many authors [1$10]$ and the references therein. We just mention some earlier work about it. Brézis and Nirenberg [1] proved that the critical problem with small linear perturbations can provide positive solutions. In [3], Rabinowitz proved the existence of standing wave solutions of nonlinear Schrödinger equations. Making a standing wave ansatz reduces the problem to that of studying a class of semilinear elliptic equations. Floer and Weinstein [10] proved that Schrödinger equation with potential $V$ and cubic nonlinearity has standing wave solutions concentrated near each nondegenerate critical point of $V$.

However, a great attention has been focused on the study of problems involving the fractional Laplacian recently. This type of operator seems to have a prevalent role in physical situations such as combustion and dislocations in mechanical systems or in crystals. In addition, these operators arise in modelling diffusion and transport in a highly heterogeneous medium. This type of problems has been studied by many authors [11-18] and the references therein.

Servadei and Valdinoci [11-14] studied the problem

$$
\begin{gathered}
L_{K} u+\lambda u+|u|^{2^{*}(s)-2} u+f(x, u)=0 \quad \text { in } \Omega, \\
u=0 \quad \text { in } \mathbb{R}^{N} \backslash \Omega,
\end{gathered}
$$

where $s \in(0,1), \Omega$ is an open bounded set of $\mathbb{R}^{N}, N>2 s$, with Lipschitz boundary, $\lambda>0$ is a real parameter, and $2^{*}(s)=2 N /(N-2 s)$ is a fractional critical Sobolev exponent. $L_{K}$ is defined as follows:

$$
\begin{array}{r}
L_{K} u(x)=\frac{1}{2} \int_{\mathbb{R}^{N}}(u(x+y)+u(x-y)-u(x)) K(y) d x d y, \\
x \in \mathbb{R}^{N} .
\end{array}
$$

Here $K: \mathbb{R}^{N} \backslash\{0\} \rightarrow(0,+\infty)$ is a function such that

$$
m K \in L^{1}\left(\mathbb{R}^{N}\right), \quad \text { where } m(x)=\min \left\{|x|^{2}, 1\right\} ;
$$

there exists $\theta>0$ such that $K(x) \geq \theta|x|^{-(N+2 s)}$ and $K(x)=$ $K(-x)$ for any $x \in \mathbb{R}^{N} \backslash\{0\}$. They proved that problem (1) admits a nontrivial solution for any $\lambda>0$. They also studied the case $f(x, u) \equiv 0$ and $K(x)=|x|^{-(N+2 s)}$, respectively.

Felmer et al. [15] studied the following nonlinear Schrödinger equation with fractional Laplacian:

$$
\begin{gathered}
(-\Delta)^{\alpha} u+u=f(x, u) \quad \text { in } \mathbb{R}^{N}, \\
u>0 \quad \text { in } \mathbb{R}^{N}, \quad \lim _{|x| \rightarrow \infty} u(x)=0,
\end{gathered}
$$

where $0<\alpha<1, N \geq 2$, and $f: \mathbb{R}^{N} \times \mathbb{R} \rightarrow \mathbb{R}$ is superlinear and has subcritical growth with respect to $u$. The 
fractional Laplacian can be characterized as $F\left((-\Delta)^{\alpha} \phi\right)(\zeta)=$ $|\zeta|^{2 \alpha} F(\phi)(\zeta)$, where $F$ is the Fourier transform. They gave the proof of existence of positive solutions and further analyzed regularity, decay, and symmetry properties of these solutions.

In this paper, we consider the following problem:

$$
\begin{array}{r}
(-\Delta)^{s} u+V(x) u=|u|^{2^{*}(s)-2} u+f(x, u) \quad \text { in } \mathbb{R}^{N}, \\
u \in H^{s}\left(\mathbb{R}^{N}\right),
\end{array}
$$

with critical exponent $2^{*}(s)=2 N /(N-2 s), N>2 s, s \in$ $(0,1)$, where $V(x) \geq 0$ has a potential well, where $(-\Delta)^{s}$ is the fractional Laplace operator, which may be defined as

$$
\begin{array}{r}
-(-\Delta)^{s} u(x)=\frac{1}{2} \int_{\mathbb{R}^{N}} \frac{u(x+y)+u(x-y)-2 u(x)}{|y|^{N+2 s}} d y, \\
x \in \mathbb{R}^{N} .
\end{array}
$$

$H^{s}\left(\mathbb{R}^{N}\right)$ is the usual fractional Sobolev space.

$f: \mathbb{R}^{N} \times \mathbb{R} \rightarrow \mathbb{R}$ is a lower order perturbation of the critical power $|u|^{2^{*}(s)-2} u$. Now we give our main assumptions. In order to find weak solutions of (5), we will assume the following general hypotheses:

(V0) $V(x)$ is a continuous nonnegative function on $\mathbb{R}^{N}$ and satisfying

$$
\lim _{|x| \rightarrow \infty} V(x)=+\infty
$$

(f0) $f: \mathbb{R}^{N} \times \mathbb{R} \rightarrow \mathbb{R}$ is a Carathéodory function;

$(f 1) \lim _{t \rightarrow o}(f(x, t) / t)=0$ uniformly in $x \in \mathbb{R}^{N}$;

$(f 2)$ there exist $a_{1}, a_{2}>0$ and $q \in\left(2,2^{*}(s)\right), 2^{*}(s)=$ $2 N /(N-2 s)$, such that

$$
|f(x, t)| \leq a_{1}+a_{2}|t|^{q-1} \quad \text { a.e. } x \in \mathbb{R}^{N}, t \in \mathbb{R}
$$

(f3) $\sup \left\{|f(x, t)|\right.$ : a.e. $\left.x \in \mathbb{R}^{N},|t| \leq M\right\}<+\infty$ for any $M>0$;

(f4) there exists $\mu>2$ such that, for all $t>0$ and a.e. $x \in$ $\mathbb{R}^{N}$

$$
0<\mu F(x, t) \leq t f(x, t),
$$

The aim of this paper is to find solutions for (5) by variational methods. For this, we give the weak formulation of (5) by the following problem:

$$
\begin{aligned}
& \int_{\mathbb{R}^{2 N}} \frac{(u(x)-u(y))(\varphi(x)-\varphi(y))}{|x-y|^{N+2 s}} d x d y \\
&+ \int_{\mathbb{R}^{N}} V(x) u(x) \varphi(x) d x \\
&= \int_{\mathbb{R}^{N}}|u(x)|^{2^{*}(s)-2} u(x) \varphi(x) d x \\
&+\int_{\mathbb{R}^{N}} f(x, u(x)) \varphi(x) d x, \quad \forall \varphi \in H^{s}\left(\mathbb{R}^{N}\right), \\
& \\
& u \in H^{s}\left(\mathbb{R}^{N}\right) .
\end{aligned}
$$

This problem represents the Euler-Lagrange equation of the function $I: H^{s}\left(\mathbb{R}^{N}\right) \rightarrow \mathbb{R}$ defined as

$$
\begin{aligned}
I(u)= & \frac{1}{2} \int_{\mathbb{R}^{2 N}} \frac{|u(x)-u(y)|^{2}}{|x-y|^{N+2 s}} d x d y \\
& +\frac{1}{2} \int_{\mathbb{R}^{N}} V(x)|u(x)|^{2} d x \\
& -\frac{1}{2^{*}(s)} \int_{\mathbb{R}^{N}}|u(x)|^{2^{*}(s)} d x \\
& -\int_{\mathbb{R}^{N}} F(x, u(x)) d x,
\end{aligned}
$$

where $F$ is defined as in $(f 4)$. Critical points of $I$ are weak solutions of (5). We will prove the existence of the critical points of the functional $I$.

It is convenient to define

$$
\begin{gathered}
S_{s}:=\inf _{u \in E \backslash\{0\}} S_{s}(u), \\
S_{r, s}:=\inf _{u \in H_{r}^{s}\left(\mathbb{R}^{N}\right) \backslash\{0\}} S_{r, s}(u),
\end{gathered}
$$

where $E$ will be defined in Section 2 and for any $u \in E \backslash\{0\}$ $S_{s}(u)$

$$
:=\frac{\int_{\mathbb{R}^{2 N}}\left(|u(x)-u(y)|^{2} /|x-y|^{N+2 s}\right) d x d y+\int_{\mathbb{R}^{N}} V(x)|u(x)|^{2} d x}{\left(\int_{\mathbb{R}^{N}}|u(x)|^{2^{*}(s)} d x\right)^{2 / 2^{*}(s)}}
$$

and $H_{r}^{s}\left(\mathbb{R}^{N}\right)$ will be defined in Section 2 and for any $u \in$ $H_{r}^{s}\left(\mathbb{R}^{N}\right) \backslash\{0\}$

$$
\begin{aligned}
& S_{r, s}(u) \\
& :=\frac{\int_{\mathbb{R}^{2 N}}\left(|u(x)-u(y)|^{2} /|x-y|^{N+2 s}\right) d x d y+\int_{\mathbb{R}^{N}}|u(x)|^{2} d x}{\left(\int_{\mathbb{R}^{N}}|u(x)|^{2^{*}(s)} d x\right)^{2 / 2^{*}(s)}} .
\end{aligned}
$$

where $F(x, t)=\int_{0}^{t} f(x, \tau) d \tau$.
Now, we give our results as follows. 
Theorem 1. Let $N>2 s, s \in(0,1), V(x)$, and $f$ satisfy $(V 0)$ and $(f 0)-(f 4)$, respectively. Then, (5) possesses at least one nontrivial solution.

We prove Theorem 1 applying the mountain pass theorem to the functional $I$. Although the Palais-Smale sequences might lose compactness in the whole space $\mathbb{R}^{N}$, we cannot apply the mountain pass theorem directly. In [15], they used a comparison argument to over this difficulty. But we will use allow it to us to over this problem related to the lack of compactness and to show that the Palais-Smale condition holds true in a suitable range related to $S_{s}$.

Theorem 2. Let $N>2 s, s \in(0,1), V(x) \equiv 1$, and $f$ satisfy $(f 0)-(f 4)$. Then, (5) possesses at least one nontrivial radial symmetric solution.

For the case $V(x) \equiv 1$, the proof of the existence of solutions is similar to the proof of Theorem 1. To prove symmetry of solutions, we consider the subspace $H_{r}^{s}\left(\mathbb{R}^{N}\right)$ of $H^{s}\left(\mathbb{R}^{N}\right) . H_{r}^{s}\left(\mathbb{R}^{N}\right)$ consists of radial symmetric functions of $H^{s}\left(\mathbb{R}^{N}\right)$.

This paper is organized as follows. In Section 2, we give some preliminary results. In Section 3, we prove the geometry and Palais-Smale condition of the functional $I$ and finish the proof of Theorem 1. In Section 4, we finish the proof of Theorem 2.

\section{Preliminary Results}

We consider the fractional Sobolev space

$$
\begin{aligned}
H^{s}\left(\mathbb{R}^{N}\right)=\{u & \in L^{2}\left(\mathbb{R}^{N}\right) \mid \int_{\mathbb{R}^{2 N}} \frac{|u(x)-u(y)|^{2}}{|x-y|^{N+2 s}} d x d y \\
& \left.+\int_{\mathbb{R}^{N}}|u(x)|^{2} d x<+\infty\right\},
\end{aligned}
$$

equipped with norm

$$
\|u\|_{H}=\left(\int_{\mathbb{R}^{2 N}} \frac{|u(x)-u(y)|^{2}}{|x-y|^{N+2 s}} d x d y+\int_{\mathbb{R}^{N}}|u(x)|^{2} d x\right)^{1 / 2} .
$$

We denote a subspace of the fractional Sobolev space $H^{s}\left(\mathbb{R}^{N}\right)$ by $E$. This subspace $E$ is defined as the completion of $C^{\infty}\left(\mathbb{R}^{N}\right)$ with respect to the norm

$$
\|u\|_{E}=\left(\int_{\mathbb{R}^{2 N}} \frac{|u(x)-u(y)|^{2}}{|x-y|^{N+2 s}} d x d y+\int_{\mathbb{R}^{N}} V(x)|u(x)|^{2} d x\right)^{1 / 2} .
$$

Obviously, $\left(E,\|\cdot\|_{E}\right)$ is a Hilbert space, with scalar product

$$
\begin{aligned}
\langle u, \varphi\rangle_{E} & =\int_{\mathbb{R}^{2 N}} \frac{(u(x)-u(y))(\varphi(x)-\varphi(y))}{|x-y|^{N+2 s}} d x d y \\
& +\int_{\mathbb{R}^{N}} V(x) u(x) \varphi(x) d x
\end{aligned}
$$

and $E$ is continuously embedded in $H^{s}\left(\mathbb{R}^{N}\right)$.

On the other hand, we also consider the subspace $H_{r}^{s}\left(\mathbb{R}^{N}\right)$ of $H^{s}\left(\mathbb{R}^{N}\right) . H_{r}^{s}\left(\mathbb{R}^{N}\right)$ consists of radial symmetric functions of $H^{s}\left(\mathbb{R}^{N}\right)$ and has the same norm with $H^{s}\left(\mathbb{R}^{N}\right)$, and its norm is denoted by $\|u\|_{H_{r}^{s}} . H_{r}^{s}\left(\mathbb{R}^{N}\right)$ is continuously embedded in $H^{s}\left(\mathbb{R}^{N}\right)$.

The following two lemmas about the fractional Sobolev space $H^{s}\left(\mathbb{R}^{N}\right)$ are proved in [15].

Lemma 3. Let $2 \leq q \leq 2^{*}(s)=2 N /(N-2 s)$; then one has

$$
\|u\|_{L^{q}\left(\mathbb{R}^{N}\right)} \leq C\|u\|_{H} \quad \forall u \in H^{s}\left(\mathbb{R}^{N}\right) .
$$

Consequently, the embedding $E \hookrightarrow L^{2^{*}(s)}\left(\mathbb{R}^{N}\right)$ and $H_{r}^{s}\left(\mathbb{R}^{N}\right) \hookrightarrow L^{2^{*}(s)}\left(\mathbb{R}^{N}\right)$ are continuous. If further $2 \leq q<$ $2^{*}(s)$ and $\Omega \subset \mathbb{R}^{N}$ is a bounded domain, then the bound sequence $\left\{u_{k}\right\} \subset H^{s}\left(\mathbb{R}^{N}\right)$ has a convergent subsequence in $L^{q}(\Omega)$.

Thanks to Lemma 3, we can define the constants $S_{s}$ and $S_{r, s}$ and get that $S_{s}>0$ and $S_{r, s}>0$.

Lemma 4. Let $N \geq 2$. Assume that $\left\{u_{k}\right\}$ is bounded in $H^{s}\left(\mathbb{R}^{N}\right)$ and it satisfies

$$
\lim _{k \rightarrow \infty} \sup _{\xi \in \mathbb{R}^{N}} \int_{B_{K}(\xi)}\left|u_{k}(x)\right|^{2} d x=0
$$

where $R>0$. Then $u_{k} \rightarrow 0$ in $L^{q}\left(\mathbb{R}^{N}\right)$ for $2<q<2^{*}(s)$.

Lemma 5. (a) If (V0) holds true, then the embedding $E \hookrightarrow$ $L^{\nu}\left(\mathbb{R}^{N}\right)$ is compact for any $v \in\left[2,2^{*}(s)\right)$.

(b) The embedding $H_{r}^{s}\left(\mathbb{R}^{N}\right) \hookrightarrow L^{q}\left(\mathbb{R}^{N}\right)$ is compact for any $q \in\left(2,2^{*}(s)\right)$.

Proof. (a) We give the proof as in [19].

Case $v=2$. We will show that $u_{n} \rightarrow 0$ strongly in $L^{2}\left(\mathbb{R}^{N}\right)$, whenever $u_{n} \rightarrow 0$ weakly in $E$. Indeed, let $C>0$ be such that $\|u\|_{E} \leq C$. Given $\varepsilon>0$, pick $R>0$ such that $V(x) \geq 2 C^{2} / \varepsilon$ for all $|x| \geq R$ and denote by $B_{R}$ the ball of radius $R$ in $\mathbb{R}^{N}$. Then we have that $u_{n} \rightarrow 0$ weakly in $H^{s}\left(B_{R}\right)$. The compact embedding $H^{s}\left(B_{R}\right) \hookrightarrow L^{2}\left(B_{R}\right)$ implies that for some natural number $n_{0}$,

$$
\int_{B_{R}}\left|u_{n}(x)\right|^{2} d x \leq \frac{\varepsilon}{2}, \quad \forall n>n_{0}
$$


On the other hand, by our choice of $R>0$, we have

$$
\begin{aligned}
& \frac{2}{\varepsilon} \int_{\mathbb{R}^{N} \backslash B_{R}}\left|u_{n}(x)\right|^{2} d x \\
& \quad \leq \frac{1}{C^{2}} \int_{\mathbb{R}^{N} \backslash B_{R}} V(x)\left|u_{n}(x)\right|^{2} d x \leq \frac{1}{C^{2}}\left\|u_{n}\right\|_{E}^{2} \leq 1 .
\end{aligned}
$$

Combining (22) and (23), we obtain that $\left\|u_{n}\right\|_{L^{2}\left(\mathbb{R}^{N}\right)}^{2}<\varepsilon$ for all $n>n_{0}$.

Case $2<v<2^{*}(s)$. Using Lemma 3, together with the interpolation inequality (where $1 / \nu=(\sigma / 2)+\left((1-\sigma) / 2^{*}(s)\right)$ ),

$$
\begin{array}{r}
\|u\|_{L^{\nu}\left(\mathbb{R}^{N}\right)} \leq\|u\|_{L^{2}\left(\mathbb{R}^{N}\right)}^{\sigma}\|u\|_{L^{2^{*}(s)}\left(\mathbb{R}^{N}\right)}^{1-\sigma}, \\
\forall u \in L^{2}\left(\mathbb{R}^{N}\right) \cap L^{2^{*}(s)}\left(\mathbb{R}^{N}\right)
\end{array}
$$

and by the fact that the embedding $E \hookrightarrow L^{2}\left(\mathbb{R}^{N}\right)$ is compact, we can obtain that the embedding $E \hookrightarrow L^{\nu}\left(\mathbb{R}^{N}\right)$ is also compact.

(b) We give the proof as in Corollary 4.7.2 of [20].

For all $x \in \mathbb{R}^{N}$, for all $R>0$, let $m(x, R)$ be the largest number of disjoint balls with radius $R$ and the centers lie on the same sphere with radius $|x|$ centered at 0 . It is easily seen that $m(x, R) \rightarrow \infty$ as $|x| \rightarrow \infty$. By definition, for all $u \in$ $L^{2}\left(\mathbb{R}^{N}\right)$ and for all $r>0$,

$$
\int_{B_{r}(x)}|u|^{2} d x \leq m(x, r)^{-1}\|u\|_{L^{2}\left(\mathbb{R}^{N}\right)}^{2} .
$$

If $\left\{u_{j}\right\}$ is a bounded sequence in $H_{r}^{s}\left(\mathbb{R}^{N}\right)$, for all $\varepsilon>0, \exists R>0$, we have

$$
\sup _{x \in \mathbb{R}^{N}}\left\{\int_{B_{r}(x)}\left|u_{j}(x)\right|^{2} d x|| x \mid \geq R\right\}<\varepsilon .
$$

We may assume that $u_{j} \rightarrow 0$ weakly in $H_{r}^{s}\left(\mathbb{R}^{N}\right)$; then, by Lemma 3, after a subsequence $\int_{B_{R+r}(0)}\left|u_{n_{j}}\right|^{2} d x \rightarrow 0$, it follows that

$$
\sup _{x \in \mathbb{R}^{N}}\left\{\int_{B_{r}(x)}\left|u_{n_{j}}(x)\right|^{2} d x|| x \mid \leq R\right\} \rightarrow 0 .
$$

By (26), (27), and Lemma 4, we have

$$
u_{n_{j}} \longrightarrow 0 \quad \text { in } L^{q}\left(\mathbb{R}^{N}\right)
$$

for $q \in\left(2,2^{*}(s)\right)$.

In [11, Lemma 6], Servadei and Valdinoci proved the following result.

Lemma 6. Assume that $f$ satisfies ( $f 0)-(f 4)$. Then, for any $\varepsilon>0$, there exists $\delta=\delta(\varepsilon)$ such that a.e. $x \in \mathbb{R}^{N}$, and for any $t \in \mathbb{R}^{N}$

$$
|f(x, t)| \leq 2 \varepsilon|t|+q \delta(\varepsilon)|t|^{q-1},
$$

and so, as a consequence,

$$
|F(x, t)| \leq \varepsilon|t|^{2}+\delta(\varepsilon)|t|^{q},
$$

where $F$ is defined as in $(f 4)$ and $q \in\left(2,2^{*}(s)\right)$.
In [17, Lemma 4], Servadei and Valdinoci proved the following result.

Lemma 7. Assume that $f$ satisfies $(f 0)-(f 4)$. Then, there exist two positive measurable functions $m=m(x)$ and $M=M(x)$ such that a.e. $x \in \mathbb{R}^{N}$, and for any $t \in \mathbb{R}$,

$$
F(x, t) \geq m(x)|t|^{\mu}-M(x)
$$

where $F$ is defined as in (f4), $2<\mu<2^{*}(s)$, and $m, M \in$ $L^{\infty}\left(\mathbb{R}^{N}\right)$.

\section{The Proof of Theorem 1}

In this section we study the mountain pass geometry and Palais-Smale condition in a suitable energy range and finish the proof of Theorem 1 . We consider the functional

$$
\begin{aligned}
I(u)= & \frac{1}{2} \int_{\mathbb{R}^{2 N}} \frac{|u(x)-u(y)|^{2}}{|x-y|^{N+2 s}} d x d y+\frac{1}{2} \int_{\mathbb{R}^{N}} V(x)|u(x)|^{2} d x \\
& -\frac{1}{2^{*}(s)} \int_{\mathbb{R}^{N}}|u(x)|^{2^{*}(s)} d x-\int_{\mathbb{R}^{N}} F(x, u(x)) d x,
\end{aligned}
$$

where $F$ is defined as in $(f 4)$.

Then $I \in C^{1}(E, \mathbb{R})$ and critical points of $I$ are solutions of

$$
\begin{array}{r}
(-\Delta)^{s} u+V(x) u=|u|^{2^{*}(s)-2} u+f(x, u), \\
u \in H^{s}\left(\mathbb{R}^{N}\right) .
\end{array}
$$

The Fréchet derivative of $I$ is

$$
\begin{aligned}
\left\langle I^{\prime}(u), \varphi\right\rangle= & \int_{\mathbb{R}^{2 N}} \frac{(u(x)-u(y))(\varphi(x)-\varphi(y))}{|x-y|^{N+2 s}} d x d y \\
& +\int_{\mathbb{R}^{N}} V(x) u(x) \varphi(x) d x \\
& -\int_{\mathbb{R}^{N}}|u(x)|^{2^{*}(s)-2} u(x) \varphi(x) d x \\
& -\int_{\mathbb{R}^{N}} f(x, u(x)) \varphi(x) d x \quad \forall \varphi \in E .
\end{aligned}
$$

Proposition 8. Let $N>2 s, s \in(0,1), V(x)$, and $f$ satisfy $(V 0)$ and $(f 0)-(f 4)$, respectively. Then, there exist $\rho>0$ and $\beta>0$ such that for any $u \in E$ with $\|u\|_{E}=\rho$ it results that $I(u) \geq \beta$. 
Proof. Let $u$ be a function in E. By Lemma 3 and (30), we get that, for any $\varepsilon>0$,

$$
\begin{aligned}
I(u) \geq & \frac{1}{2} \int_{\mathbb{R}^{2 N}} \frac{|u(x)-u(y)|^{2}}{|x-y|^{N+2 s}} d x d y+\frac{1}{2} \int_{\mathbb{R}^{N}} V(x)|u(x)|^{2} d x \\
& -\frac{1}{2^{*}(s)} \int_{\mathbb{R}^{N}}|u(x)|^{2^{*}(s)} d x-\varepsilon \int_{\mathbb{R}^{N}}|u(x)|^{2} d x \\
& -\delta(\varepsilon) \int_{\mathbb{R}^{N}}|u(x)|^{q} d x \\
= & \frac{1}{2}\|u\|_{E}^{2}-\varepsilon\|u\|_{L^{2}\left(\mathbb{R}^{N}\right)}^{2}-\frac{1}{2^{*}(s)}\|u\|_{L^{2^{*}(s)}\left(\mathbb{R}^{N}\right)}^{2^{*}(s)} \\
& -\delta(\varepsilon)\|u\|_{L^{q}\left(\mathbb{R}^{N}\right)}^{q} \\
\geq & \frac{1}{2}\|u\|_{E}^{2}-\varepsilon C_{1}\|u\|_{E}^{2}-\frac{1}{2^{*}(s)} C_{2}\|u\|_{E}^{2^{*}(s)}-\delta(\varepsilon) C_{3}\|u\|_{E}^{q} .
\end{aligned}
$$

Choosing $\varepsilon>0$ such that $(1 / 2)-\varepsilon C_{1}>0$, by (35), it easily follows that

$$
I(u) \geq C_{4}\|u\|_{E}^{2}\left(1-C_{5}\|u\|_{E}^{2^{*}(s)-2}-C_{6}\|u\|_{E}^{q-2}\right),
$$

for suitable positive constants $C_{4}, C_{5}$, and $C_{6}$.

Now, let $u \in E$ be such that $\|u\|_{E}=\rho>0$. Since $2^{*}(s)>$ $q>2$, we can choose $\rho$ sufficiently small, so that

$$
\inf _{u \in E,\|u\|_{E}=\rho} I(u) \geq C_{4} \rho^{2}\left(1-C_{5} \rho^{2^{*}(s)-2}-C_{6} \rho^{q-2}\right)=: \beta>0 .
$$

Hence, Proposition 8 is proved.

Proposition 9. There exists $u_{0} \in E \backslash\{0\}$ with $u_{0} \geq 0$ a.e. in $\mathbb{R}^{N}$, such that

$$
\sup _{t \geq 0} I\left(t u_{0}\right)<\frac{s}{N} S_{s}^{N / 2 s}
$$

Proof. By definition of $S_{s}$, we have that there exists $u_{0} \in E \backslash\{0\}$ such that

$$
\frac{\left\|u_{0}\right\|_{E}^{2}}{\left\|u_{0}\right\|_{L^{2^{*}(s)}\left(\mathbb{R}^{N}\right)}^{2}}<S_{s}+\varepsilon,
$$

for any $\varepsilon>0$. We have $u_{0} \geq 0$ a.e. in $\mathbb{R}^{N}$, or else we can take $\left|u_{0}\right| \in E$. Indeed, by triangle inequality, a.e. $x, y \in \mathbb{R}^{N}$,

$$
|| u_{0}(x)|-| u_{0}(y)|| \leq\left|u_{0}(x)-u_{0}(y)\right|
$$

and so

$$
\left\|\left|u_{0}\right|\right\|_{E}^{2} \leq\left\|u_{0}\right\|_{E}^{2}
$$

Thus,

$$
\frac{\left\|u_{0} \mid\right\|_{E}^{2}}{\left\|u_{0}\right\|_{L^{2^{*}(s)}\left(\mathbb{R}^{N}\right)}^{2}}<S_{s}+\varepsilon .
$$

It follows that there exists $t_{0}>0$ such that

$$
\begin{aligned}
\max _{t \geq 0} I\left(t u_{0}\right) & \\
= & \max _{t \geq 0}\left(\frac{t^{2}}{2}\left\|u_{0}\right\|_{E}^{2}-\frac{t^{2^{*}(s)}}{2^{*}(s)}\left\|u_{0}\right\|_{L^{2^{*}(s)}\left(\mathbb{R}^{N}\right)}^{2^{*}(s)}\right. \\
& \left.\quad-\int_{\mathbb{R}^{N}} F\left(x, t u_{0}(x)\right) d x\right) \\
= & \frac{t_{0}^{2}}{2}\left\|u_{0}\right\|_{E}^{2}-\frac{t_{0}^{2^{*}(s)}}{2^{*}(s)}\left\|u_{0}\right\|_{L^{2^{*}(s)}\left(\mathbb{R}^{N}\right)}^{2^{*}(s)}-\int_{\mathbb{R}^{N}} F\left(x, t_{0} u_{0}(x)\right) d x \\
\leq & \max _{t \geq 0}\left(\frac{t^{2}}{2}\left\|u_{0}\right\|_{E}^{2}-\frac{t^{2^{*}(s)}}{2^{*}(s)}\left\|u_{0}\right\|_{L^{2^{*}(s)}\left(\mathbb{R}^{N}\right)}^{2^{*}(s)}\right) \\
& -\int_{\mathbb{R}^{N}} F\left(x, t_{0} u_{0}(x)\right) d x \\
= & \frac{s}{N}\left(\frac{\left\|u_{0}\right\|_{E}^{2}}{\left\|u_{0}\right\|_{L^{2^{*}(s)}\left(\mathbb{R}^{N}\right)}^{2}}\right)^{N / 2 s}-\int_{\mathbb{R}^{N}} F\left(x, t_{0} u_{0}(x)\right) d x \\
< & \frac{s}{N}\left(S_{s}+\varepsilon\right)^{N / 2 s}-\int_{\mathbb{R}^{N}} F\left(x, t_{0} u_{0}(x)\right) d x,
\end{aligned}
$$

thanks to (39). Since $\int_{\mathbb{R}^{N}} F\left(x, t_{0} u_{0}(x)\right) d x>0$, so we can choose $\varepsilon>0$ such that

$$
\sup _{t \geq 0} I\left(t u_{0}\right)<\frac{s}{N} S_{s}^{N / 2 s}
$$

Hence, Proposition 9 is proved.

Proposition 10. Let $N>2 s, s \in(0,1), V(x)$, and $f$ satisfy $(V 0)$ and ( $f 0)-(f 4)$, respectively. Then, there exists $e \in E$ such that $e \geq 0$ a.e. in $\mathbb{R}^{N},\|e\|_{E}>\rho$, and $I(e)<\beta$, where $\rho$ and $\beta$ are given in Proposition 8.

In particular, we can construct $e$ as follows:

$$
e=t_{0} u_{0}
$$

with $u_{0}$ as in (38) and $t_{0}>0$ large enough.

Proof. We fix $u \in E$ such that $\|u\|_{E} \neq 0$. By (31), we get

$$
\begin{aligned}
I(t u) \leq & \frac{1}{2} t^{2} \int_{\mathbb{R}^{2 N}} \frac{|u(x)-u(y)|^{2}}{|x-y|^{N+2 s}} d x d y \\
& +\frac{1}{2} t^{2} \int_{\mathbb{R}^{N}} V(x)|u(x)|^{2} d x \\
& -\frac{1}{2} t^{2^{*}(s)} \int_{\mathbb{R}^{N}}|u(x)|^{2^{*}(s)} d x \\
& -t^{\mu} \int_{\mathbb{R}^{N}} m(x)|u(x)|^{\mu}-M(x) d x \\
= & \frac{1}{2} t^{2}\|u\|_{E}^{2}-\frac{1}{2^{*}(s)} t^{2^{*}(s)}\|u\|_{L^{2^{*}(s)}\left(\mathbb{R}^{N}\right)}^{2^{*}(s)} \\
& -t^{\mu} C_{1}\|u\|_{L^{\mu}\left(\mathbb{R}^{N}\right)}^{\mu}+C_{2},
\end{aligned}
$$

where $C_{1}, C_{2} \geq 0$ are constant. Since $2^{*}(s)>2$ and $\mu>2$, passing to the limit as $t \rightarrow+\infty$, we get that $I(t u) \rightarrow-\infty$, 
so that the assertion follows taking $e=t u$, with $t$ sufficiently large.

In particular, we can take $u=u_{0} \in E$, then $e=t_{0} u_{0}$ with $t_{0}$ large enough.

We easily see that $I(0)=0<\beta$, with $\beta$ given in Proposition 8. Now, set

$$
c=\inf _{T \in \Gamma} \sup _{u \in T([0,1])} I(u)
$$

where

$$
\Gamma=\{T \in C([0,1] ; E): T(0)=0, T(1)=e\},
$$

with $e=t_{0} u_{0}$ in Proposition 9.

In [11, the proof of Theorem 1], Servadei and Valdinoci proved the following result.

Proposition 11. The constant $c$ is given in (47) such that

$$
\beta \leq c<\frac{s}{N} S_{s}^{N / 2 s},
$$

where $\beta$ is given in Proposition 8 and $S_{s}$ is defined in formula (12).

By [6, Theorem 2.2], we have a sequence $u_{j}$ in $E$ such that

$$
\begin{gathered}
I\left(u_{j}\right) \longrightarrow c, \\
\sup \left\{\left|\left\langle I^{\prime}\left(u_{j}\right), \varphi\right\rangle\right|: \varphi \in E,\|\varphi\|_{E}=1\right\} \longrightarrow 0
\end{gathered}
$$

as $j \rightarrow+\infty$.

Proposition 12. There exists $u_{\infty} \in E$ such that, up to a subsequence, $\left\|u_{j}-u_{\infty}\right\|_{E} \rightarrow 0$ as $j \rightarrow+\infty$.

Proof. We proceed by steps.

Step 1. The sequence $u_{j}$ is bounded in $E$.

Proof. For any $j \in \mathbb{N}$ by (50) and (51) it easily follows that there exists $C_{1}>0$ such that

$$
\begin{gathered}
\left|I\left(u_{j}\right)\right| \leq C_{1}, \\
\left|\left\langle I^{\prime}\left(u_{j}\right), \frac{u_{j}}{\left\|u_{j}\right\|_{E}}\right\rangle\right| \leq C_{1} .
\end{gathered}
$$

As a consequence of (52), we have

$$
I\left(u_{j}\right)-\frac{1}{\mu}\left\langle I^{\prime}\left(u_{j}\right), u_{j}\right\rangle \leq C_{1}\left(1+\left\|u_{j}\right\|_{E}\right) .
$$

By $(f 2)$ and $(f 4)$, we have $\mu<2^{*}(s)$ and

$$
\begin{aligned}
& I\left(u_{j}\right)- \frac{1}{\mu}\left\langle I^{\prime}\left(u_{j}\right), u_{j}\right\rangle \\
&=\left(\frac{1}{2}-\frac{1}{\mu}\right)\left(\int_{\mathbb{R}^{2 N}} \frac{\left|u_{j}(x)-u_{j}(y)\right|^{2}}{|x-y|^{N+2 s}} d x d y\right. \\
&\left.\quad+\int_{\mathbb{R}^{N}} V(x)\left|u_{j}(x)\right|^{2} d x\right)
\end{aligned}
$$

$$
\begin{aligned}
& +\left(\frac{1}{\mu}-\frac{1}{2^{*}(s)}\right) \int_{\mathbb{R}^{N}}\left|u_{j}(x)\right|^{2^{*}(s)} d x \\
& -\int_{\mathbb{R}^{N}} F\left(x, u_{j}(x)\right) d x \\
& +\frac{1}{\mu} \int_{\mathbb{R}^{N}} f\left(x, u_{j}(x)\right) u_{j}(x) d x \\
& \geq\left(\frac{1}{2}-\frac{1}{\mu}\right)\left\|u_{j}\right\|_{E}^{2}+\left(\frac{1}{\mu}-\frac{1}{2^{*}(s)}\right)\left\|u_{j}\right\|_{L^{2^{*}(s)}\left(\mathbb{R}^{N}\right)} \cdot
\end{aligned}
$$

We obtain that $u_{j}$ is bounded in $E$ by $2<\mu<2^{*}(s)$, (53), and (54).

Step 2. Problem (10) admits a solution $u_{\infty} \in E$.

Proof. By Step 1 and since $E$ is a reflexive space, up to a subsequence, still denoted by $u_{j}$, there exists $u_{\infty} \in E$ such that $u_{j} \rightarrow u_{\infty}$ weakly in $E$; that is,

$$
\begin{aligned}
\int_{\mathbb{R}^{2 N}} & \frac{\left(u_{j}(x)-u_{j}(y)\right)(\varphi(x)-\varphi(y))}{|x-y|^{N+2 s}} d x d y \\
& +\int_{\mathbb{R}^{N}} V(x) u_{j}(x) \varphi(x) d x \\
\longrightarrow & \int_{\mathbb{R}^{2 N}} \frac{\left(u_{\infty}(x)-u_{\infty}(y)\right)(\varphi(x)-\varphi(y))}{|x-y|^{N+2 s}} d x d y \\
& +\int_{\mathbb{R}^{N}} V(x) u_{\infty}(x) \varphi(x) d x
\end{aligned}
$$

as $j \rightarrow+\infty$. By $2<\mu<2^{*}(s),(53)$, and (54), we have that $u_{j}$ is bounded in $L^{2^{*}(s)}\left(\mathbb{R}^{N}\right)$. Since $L^{2^{*}(s)}\left(\mathbb{R}^{N}\right)$ is a reflexive space, up to a subsequence,

$$
u_{j} \longrightarrow u_{\infty} \quad \text { weakly in } L^{2^{*}(s)}\left(\mathbb{R}^{N}\right)
$$

as $j \rightarrow+\infty$, while by Lemma 5(a), up to a subsequence,

$$
\begin{gathered}
u_{j} \longrightarrow u_{\infty} \quad \text { in } L^{\nu}\left(\mathbb{R}^{N}\right) \\
u_{j} \longrightarrow u_{\infty} \quad \text { a.e. in } \mathbb{R}^{N}
\end{gathered}
$$

as $j \rightarrow+\infty$, for any $v \in\left[2,2^{*}(s)\right)$. By (56) and since $\left|u_{j}\right|^{2^{*}(s)-2} u_{j}$ is bounded in $L^{2^{*}(s) /\left(2^{*}(s)-1\right)}\left(\mathbb{R}^{N}\right)$, we have

$\left|u_{j}\right|^{2^{*}(s)-2} u_{j} \longrightarrow\left|u_{\infty}\right|^{2^{*}(s)-2} u_{\infty} \quad$ weakly in $L^{2^{*}(s) /\left(2^{*}(s)-1\right)}\left(\mathbb{R}^{N}\right)$

as $j \rightarrow+\infty$. By the proof of Lemma 6 [11, Lemma 6], we get

$$
\begin{aligned}
& \int_{\mathbb{R}^{N}} f(x, u(x)) d x \\
& \quad \leq 2 \varepsilon \int_{B_{\sigma}}|u(x)| d x+q \delta(\varepsilon) \int_{\mathbb{R}^{N} \backslash B_{\sigma}}|u(x)|^{q-1} d x .
\end{aligned}
$$


Moreover, we have, by taking $\varepsilon=1$,

$$
\int_{\mathbb{R}^{N}} f(x, u(x)) d x \leq C_{1}+C_{2} \int_{\mathbb{R}^{N} \backslash B_{\sigma}}|u(x)|^{q-1} d x .
$$

By (61), $u_{j}$ being bounded in $E$, and Lemma 5(a), we obtain

$$
f\left(\cdot, u_{j}(\cdot)\right) \text { is bounded in } L^{q /(q-1)}\left(\mathbb{R}^{N}\right) .
$$

Since $L^{q /(q-1)}\left(\mathbb{R}^{N}\right)$ is a reflexive space, we get

$$
f\left(\cdot, u_{j}(\cdot)\right) \longrightarrow f\left(\cdot, u_{\infty}(\cdot)\right) \quad \text { weakly in } L^{q /(q-1)}\left(\mathbb{R}^{N}\right)
$$

as $j \rightarrow+\infty$. It is easily seen that

$$
\begin{array}{r}
\int_{\mathbb{R}^{N}} f\left(x, u_{j}(x)\right) \varphi(x) d x \longrightarrow \int_{\mathbb{R}^{N}} f\left(x, u_{\infty}(x)\right) \varphi(x) d x \\
\forall \varphi \in L^{q}\left(\mathbb{R}^{N}\right)
\end{array}
$$

as $j \rightarrow+\infty$ and so, in particular,

$$
\begin{array}{r}
\int_{\mathbb{R}^{N}} f\left(x, u_{j}(x)\right) \varphi(x) d x \longrightarrow \int_{\mathbb{R}^{N}} f\left(x, u_{\infty}(x)\right) \varphi(x) d x \\
\forall \varphi \in E
\end{array}
$$

as $j \rightarrow+\infty$. Since (51) holds true, for any $\varphi \in E$,

$$
\begin{aligned}
0 & \longleftarrow\left\langle I^{\prime}\left(u_{j}\right), \varphi\right\rangle \\
= & \int_{\mathbb{R}^{2 N}} \frac{\left(u_{j}(x)-u_{j}(y)\right)(\varphi(x)-\varphi(y))}{|x-y|^{N+2 s}} d x d y \\
& +\int_{\mathbb{R}^{N}} V(x) u_{j}(x) \varphi(x) d x \\
& -\int_{\mathbb{R}^{N}}\left|u_{j}(x)\right|^{2^{*}(s)-2} u_{j}(x) \varphi(x) d x \\
& -\int_{\mathbb{R}^{N}} f\left(x, u_{j}(x)\right) \varphi(x) d x .
\end{aligned}
$$

Passing to the limit in this expression as $j \rightarrow+\infty$ and taking into account (55), (57), (59), and (65), we get

$$
\begin{aligned}
\int_{\mathbb{R}^{2 N}} & \frac{\left(u_{\infty}(x)-u_{\infty}(y)\right)(\varphi(x)-\varphi(y))}{|x-y|^{N+2 s}} d x d y \\
& +\int_{\mathbb{R}^{N}} V(x) u_{\infty}(x) \varphi(x) d x \\
& -\int_{\mathbb{R}^{N}}\left|u_{\infty}(x)\right|^{2^{*}(s)-2} u_{\infty}(x) \varphi(x) d x \\
& -\int_{\mathbb{R}^{N}} f\left(x, u_{\infty}(x)\right) \varphi(x) d x=0
\end{aligned}
$$

for any $\varphi \in E$; that is, $u_{\infty}$ is a solution of problem (10).
Step 3. The following equality holds true:

$$
\begin{aligned}
I\left(u_{\infty}\right)= & \frac{s}{N} \int_{\mathbb{R}^{N}}\left|u_{\infty}(x)\right|^{2^{*}(s)} d x \\
& +\frac{1}{2} \int_{\mathbb{R}^{N}} f\left(x, u_{\infty}(x)\right) u_{\infty}(x) d x \\
& -\int_{\mathbb{R}^{N}} F\left(x, u_{\infty}(x)\right) d x \geq 0 .
\end{aligned}
$$

Proof. By Step 2, taking $\varphi=u_{\infty} \in E$ as a test function in (10), we have

$$
\begin{array}{r}
\int_{\mathbb{R}^{2 N}} \frac{\left|u_{\infty}(x)-u_{\infty}(y)\right|^{2}}{|x-y|^{N+2 s}} d x d y+\int_{\mathbb{R}^{N}} V(x)\left|u_{\infty}(x)\right|^{2} d x \\
\quad=\int_{\mathbb{R}^{N}}\left|u_{\infty}(x)\right|^{2^{*}(s)} d x+\int_{\mathbb{R}^{N}} f\left(x, u_{\infty}(x)\right) u_{\infty}(x) d x
\end{array}
$$

so that

$$
\begin{aligned}
I\left(u_{\infty}\right)= & \frac{s}{N} \int_{\mathbb{R}^{N}}\left|u_{\infty}(x)\right|^{2^{*}(s)} d x \\
& +\frac{1}{2} \int_{\mathbb{R}^{N}} f\left(x, u_{\infty}(x)\right) u_{\infty}(x) d x \\
& -\int_{\mathbb{R}^{N}} F\left(x, u_{\infty}(x)\right) d x \geq 0 .
\end{aligned}
$$

The last inequality follows from assumption $(f 4)$.

Now, we conclude the proof of Proposition 12.

We write $v_{j}:=u_{j}-u_{\infty}$; then, $v_{j} \rightarrow 0$ weakly in $E$. Moreover, since (58) holds true, by the Brézis-Lieb Lemma, we get

$$
\begin{aligned}
\int_{\mathbb{R}^{2 N}} & \frac{\left|u_{j}(x)-u_{j}(y)\right|^{2}}{|x-y|^{N+2 s} d x d y} \\
& +\int_{\mathbb{R}^{N}} V(x)\left|u_{j}(x)\right|^{2} d x \\
= & \int_{\mathbb{R}^{2 N}} \frac{\left|v_{j}(x)-v_{j}(y)\right|^{2}}{|x-y|^{N+2 s} d x d y} \\
& +\int_{\mathbb{R}^{N}} V(x)\left|v_{j}(x)\right|^{2} d x \\
& +\int_{\mathbb{R}^{2 N}} \frac{\left|u_{\infty}(x)-u_{\infty}(y)\right|^{2}}{|x-y|^{N+2 s}} d x d y \\
& +\int_{\mathbb{R}^{N}} V(x)\left|u_{\infty}(x)\right|^{2} d x+\circ(1), \\
\int_{\mathbb{R}^{N}}\left|u_{j}(x)\right|^{2^{*}(s)} d x= & \int_{\mathbb{R}^{N}}\left|v_{j}(x)\right|^{2^{*}(s)} d x \\
+ & \int_{\mathbb{R}^{N}}\left|u_{\infty}(x)\right|^{2^{*}(s)} d x+\circ(1) .
\end{aligned}
$$


Then,

$$
\begin{aligned}
& c \longleftarrow I\left(u_{j}\right)=\frac{1}{2} \int_{\mathbb{R}^{2 N}} \frac{\left|u_{j}(x)-u_{j}(y)\right|^{2}}{|x-y|^{N+2 s}} d x d y \\
& +\frac{1}{2} \int_{\mathbb{R}^{N}} V(x)\left|u_{j}(x)\right|^{2} d x \\
& -\frac{1}{2^{*}(s)} \int_{\mathbb{R}^{N}}\left|u_{j}(x)\right|^{2^{*}(s)} d x \\
& -\int_{\mathbb{R}^{N}} F\left(x, u_{j}(x)\right) d x \\
& =\frac{1}{2} \int_{\mathbb{R}^{2 N}} \frac{\left|v_{j}(x)-v_{j}(y)\right|^{2}}{|x-y|^{N+2 s}} d x d y \\
& +\frac{1}{2} \int_{\mathbb{R}^{N}} V(x)\left|v_{j}(x)\right|^{2} d x \\
& +\frac{1}{2} \int_{\mathbb{R}^{2 N}} \frac{\left|u_{\infty}(x)-u_{\infty}(y)\right|^{2}}{|x-y|^{N+2 s}} d x d y \\
& +\frac{1}{2} \int_{\mathbb{R}^{N}} V(x)\left|u_{\infty}(x)\right|^{2} d x \\
& -\frac{1}{2^{*}(s)} \int_{\mathbb{R}^{N}}\left|v_{j}(x)\right|^{2^{*}(s)} d x \\
& -\frac{1}{2^{*}(s)} \int_{\mathbb{R}^{N}}\left|u_{\infty}(x)\right|^{2^{*}(s)} d x \\
& -\int_{\mathbb{R}^{N}} F\left(x, u_{\infty}(x)\right) d x+\circ(1) \\
& =I\left(u_{\infty}\right)+\frac{1}{2} \int_{\mathbb{R}^{2 N}} \frac{\left|v_{j}(x)-v_{j}(y)\right|^{2}}{|x-y|^{N+2 s}} d x d y \\
& +\frac{1}{2} \int_{\mathbb{R}^{N}} V(x)\left|v_{j}(x)\right|^{2} d x \\
& -\frac{1}{2^{*}(s)} \int_{\mathbb{R}^{N}}\left|v_{j}(x)\right|^{2^{*}(s)} d x+\circ(1), \\
& \left\langle I^{\prime}\left(u_{j}\right), u_{j}\right\rangle=\int_{\mathbb{R}^{2 N}} \frac{\left|u_{j}(x)-u_{j}(y)\right|^{2}}{|x-y|^{N+2 s}} d x d y \\
& +\int_{\mathbb{R}^{N}} V(x)\left|u_{j}(x)\right|^{2} d x \\
& -\int_{\mathbb{R}^{N}}\left|u_{j}(x)\right|^{2^{*}(s)} d x \\
& -\int_{\mathbb{R}^{N}} f\left(x, u_{j}(x)\right) u_{j}(x) d x \\
& =\int_{\mathbb{R}^{2 N}} \frac{\left|v_{j}(x)-v_{j}(y)\right|^{2}}{|x-y|^{N+2 s}} d x d y \\
& +\int_{\mathbb{R}^{N}} V(x)\left|v_{j}(x)\right|^{2} d x
\end{aligned}
$$

$$
\begin{aligned}
& +\int_{\mathbb{R}^{2 N}} \frac{\left|u_{\infty}(x)-u_{\infty}(y)\right|^{2}}{|x-y|^{N+2 s}} d x d y \\
& +\int_{\mathbb{R}^{N}} V(x)\left|u_{\infty}(x)\right|^{2} d x-\int_{\mathbb{R}^{N}}\left|v_{j}(x)\right|^{2^{*}(s)} d x \\
& -\int_{\mathbb{R}^{N}}\left|u_{\infty}(x)\right|^{2^{*}(s)} d x \\
& -\int_{\mathbb{R}^{N}} f\left(x, u_{\infty}(x)\right) u_{\infty}(x) d x+\circ(1) \\
& =\left\langle I^{\prime}\left(u_{\infty}\right), u_{\infty}\right\rangle+\int_{\mathbb{R}^{2 N}} \frac{\left|v_{j}(x)-v_{j}(y)\right|^{2}}{|x-y|^{N+2 s}} d x d y \\
& +\int_{\mathbb{R}^{N}} V(x)\left|v_{j}(x)\right|^{2} d x \\
& -\int_{\mathbb{R}^{N}}\left|v_{j}(x)\right|^{2^{*}(s)} d x+\circ(1) .
\end{aligned}
$$

$\operatorname{By}\left\langle I^{\prime}\left(u_{\infty}\right), u_{\infty}\right\rangle=0$ and $\left\langle I^{\prime}\left(u_{j}\right), u_{j}\right\rangle \rightarrow 0$, we get

$$
\begin{gathered}
\int_{\mathbb{R}^{2 N}} \frac{\left|v_{j}(x)-v_{j}(y)\right|^{2}}{|x-y|^{N+2 s}} d x d y+\int_{\mathbb{R}^{N}} V(x)\left|v_{j}(x)\right|^{2} d x \longrightarrow b, \\
\int_{\mathbb{R}^{N}}\left|v_{j}(x)\right|^{2^{*}(s)} d x \longrightarrow b .
\end{gathered}
$$

By the definition of $S_{s}$, we have

$$
\left\|v_{j}\right\|_{E}^{2} \geq S_{s}\left\|v_{j}\right\|_{L^{2^{*}(s)}\left(\mathbb{R}^{N}\right)}^{2}
$$

and so $b \geq S_{s} b^{2 / 2^{*}(s)}$. Either $b=0$ or $b \geq S_{s}^{N / 2 s}$. If $b=0$, the proof is complete. Assuming that $b \geq S_{s}^{N / 2 s}$, we obtain, from (49), (68), and (72),

$$
\frac{s}{N} S_{s}^{N / 2 s} \leq\left(\frac{1}{2}-\frac{1}{2^{*}(s)}\right) b \leq c<\frac{s}{N} S_{s}^{N / 2 s}
$$

which is a contradiction. Thus $b=0$ and

$$
\left\|u_{j}-u_{\infty}\right\|_{E} \longrightarrow 0
$$

as $j \rightarrow+\infty$. This ends the proof of Proposition 12 .

We have finished the proof of Theorem 1 by Propositions 8,10 , and 12 and the mountain pass theorem.

\section{The Proof of Theorem 2}

In this section we consider the case $V(x) \equiv 1$, study the mountain pass geometry and Palais-Smale condition in a 
suitable energy range, and finish the proof of Theorem 2. We consider the functional

$$
\begin{aligned}
I_{1}(u)= & \frac{1}{2} \int_{\mathbb{R}^{2 N}} \frac{|u(x)-u(y)|^{2}}{|x-y|^{N+2 s}} d x d y+\frac{1}{2} \int_{\mathbb{R}^{N}}|u(x)|^{2} d x \\
& -\frac{1}{2^{*}(s)} \int_{\mathbb{R}^{N}}|u(x)|^{2^{*}(s)} d x-\int_{\mathbb{R}^{N}} F(x, u(x)) d x,
\end{aligned}
$$

where $F$ is defined as in $(f 4)$. Then $I_{1} \in C^{1}(E, \mathbb{R})$ and critical points of $I_{1}$ are solutions of

$$
(-\Delta)^{s} u+u=|u|^{2^{*}(s)-2} u+f(x, u), \quad u \in H^{s}\left(\mathbb{R}^{N}\right) .
$$

The Fréchet derivative of $I_{1}$ is

$$
\begin{aligned}
\left\langle I_{1}^{\prime}(u), \varphi\right\rangle= & \int_{\mathbb{R}^{2 N}} \frac{(u(x)-u(y))(\varphi(x)-\varphi(y))}{|x-y|^{N+2 s}} d x d y \\
& +\int_{\mathbb{R}^{N}} u(x) \varphi(x) d x \\
& -\int_{\mathbb{R}^{N}}|u(x)|^{2^{*}(s)-2} u(x) \varphi(x) d x \\
& -\int_{\mathbb{R}^{N}} f(x, u(x)) \varphi(x) d x
\end{aligned}
$$

for any $\varphi \in H_{r}^{s}\left(\mathbb{R}^{N}\right)$.

Similar to the proof of Theorem 1, we have the following conclusions.

Proposition 13. Let $N>2 s, s \in(0,1)$, and $f$ satisfy $(f 0)-$ ( $f 4)$. Then, there exist $\rho>0$ and $\beta>0$ such that for any $u \in H_{r}^{s}\left(\mathbb{R}^{N}\right)$ with $\|u\|_{H_{r}^{s}}=\rho$ it results that $I_{1}(u) \geq \beta$.

Proposition 14. There exists $u_{0} \in H_{r}^{s}\left(\mathbb{R}^{N}\right) \backslash\{0\}$ with $u_{0} \geq 0$ a.e. in $\mathbb{R}^{N}$, such that

$$
\sup _{t \geq 0} I_{1}\left(t u_{0}\right)<\frac{s}{N} S_{r, s}^{N / 2 s}
$$

Proposition 15. Let $N>2 s, s \in(0,1)$, and $f$ satisfy ( $f 0)$ (f4). Then, there exists $e \in H_{r}^{s}\left(\mathbb{R}^{N}\right)$ such that $e \geq 0$ a.e. in $\mathbb{R}^{N}$, $\|e\|_{H_{r}^{s}}>\rho$, and $I_{1}(e)<\beta$, where $\rho$ and $\beta$ are given in Proposition 13.

In particular, we can construct $e$ as follows:

$$
e=t_{0} u_{0}
$$

with $u_{0}$ as in (81) and $t_{0}>0$ large enough.

We easily see that $I_{1}(0)=0<\beta$, with $\beta$ given in Proposition 13. Now, set

$$
c=\inf _{T \in \Gamma} \sup _{u \in T([0,1])} I_{1}(u),
$$

where

$$
\Gamma=\left\{T \in C\left([0,1] ; H_{r}^{s}\left(\mathbb{R}^{N}\right)\right): T(0)=0, T(1)=e\right\},
$$

with $e=t_{0} u_{0}$ in Proposition 13

Proposition 16. The constant $c$ is given in (83) such that

$$
\beta \leq c<\frac{s}{N} S_{r, s}^{N / 2 s},
$$

where $\beta$ is given in Proposition 13 and $S_{r, s}$ is defined in formula (13).

By $\left[6\right.$, Theorem 2.2], we have a sequence $u_{j}$ in $H_{r}^{s}\left(\mathbb{R}^{N}\right)$ such that

$$
\begin{gathered}
I_{1}\left(u_{j}\right) \longrightarrow c \\
\sup \left\{\left|\left\langle I_{1}^{\prime}\left(u_{j}\right), \varphi\right\rangle\right|: \varphi \in H_{r}^{s}\left(\mathbb{R}^{N}\right),\|\varphi\|_{H_{r}^{s}}=1\right\} \rightarrow 0
\end{gathered}
$$

as $j \rightarrow+\infty$.

Proposition 17. There exists $u_{\infty} \in H_{r}^{s}\left(\mathbb{R}^{N}\right)$ such that, up to a subsequence, $\left\|u_{j}-u_{\infty}\right\|_{H_{r}^{s}} \rightarrow 0$ as $j \rightarrow+\infty$.

We have finished the proof of Theorem 2 by Propositions 13,15 , and 17 and the mountain pass theorem.

\section{Conflict of Interests}

The authors declare that there is no conflict of interests regarding the publication of this paper.

\section{Acknowledgment}

The work is supported by the National Nature Science Foundation of China (11271331).

\section{References}

[1] H. Brézis and L. Nirenberg, "Positive solutions of nonlinear elliptic equations involving critical Sobolev exponents," Communications on Pure and Applied Mathematics, vol. 36, no. 4, pp. 437-477, 1983.

[2] M. Clapp and Y. Ding, "Positive solutions of a Schrödinger equation with critical nonlinearity," Zeitschrift fur Angewandte Mathematik und Physik, vol. 55, no. 4, pp. 592-605, 2004.

[3] P. H. Rabinowitz, "On a class of nonliear Schrödinger equations," Zeitschrift für Angewandte Mathematik und Physik, vol. 43, no. 2, pp. 270-291, 1992.

[4] M. B. Yang and Y. H. Ding, "Existence of solutions for singularly perturbed Schrödinger equations with nonlocal part," Communications on Pure and Applied Analysis, vol.12, no. 2, pp. 771-783, 2013.

[5] M. B. Yang, "Existence of solutions for a quasilinear Schrödinger equation with subcritical nonlinearities," Nonlinear Analysis: Theory, Methods \& Applications, vol. 75, no. 13, pp. 5362-5373, 2012. 
[6] H. Brézis and L. Nirenberg, "Positive solutions of nonlinear elliptic equations involving critical Sobolev exponents," Communications on Pure and Applied Analysis, vol. 36, no. 4, pp. 437-477, 1983.

[7] W. A. Strauss, "Existence of solitary waves in higher dimensions," Communications in Mathematical Physics, vol. 55, no. 2, pp. 149-162, 1977.

[8] J. Chabrowski and J. Yang, "Existence theorems for the Schrödinger equation involving a critical Sobolev exponent," Zeitschrift fur Angewandte Mathematik und Physik, vol. 49, no. 2, pp. 276-293, 1998.

[9] V. Benci and G. Cerami, "Existence of positive solutions of the equation $-\Delta u+a(x) u=|u|^{(N+2) /(N-2)}$ in $\mathbb{R}^{N}$," Journal of Functional Analysis, vol. 88, no. 1, pp. 90-117, 1990.

[10] A. Floer and A. Weinstein, "Nonspreading wave packets for the cubic Schrödinger equation with a bounded potential," Journal of Functional Analysis, vol. 69, no. 3, pp. 397-408, 1986.

[11] R. Servadei and E. Valdinoci, "The Brezis-Nirenberg result forthe fractional Laplacian," Transactions of the American Mathematical Society. In press.

[12] R. Servadei and E. Valdinoci, "Fractional Laplacian equations with critical sobolev exponent," 2012, http://www.ma.utexas .edu/mp-arc-bin/mpa_yn=12-58.

[13] R. Servadei, "The Yamabe equation in a non-local setting," Advances in Nonlinear Analysis, vol. 2, no. 3, pp. 235-270, 2013.

[14] R. Servadei and A. Brézis-Nirenberg, "A Brezis-Nirenberg result for non-local critical equations in low dimension," Communications on Pure and Applied Analysis, vol. 12, no. 6, pp. 2445-2464, 2013.

[15] P. Felmer, A. Quaas, and J. G. Tan, "Positive solutions of nonlinear Schrödinger equation with fractional Laplacian," Proceedings of the Royal Society of Edinburgh, vol. 142, no. 6, pp. 1237-1262, 2012.

[16] E. Di Nezza, G. Palatucci, and E. Valdinoci, "Hitchhiker's guide to the fractional Sobolev spaces," Bulletin des Sciences Mathematiques, vol. 136, no. 5, pp. 521-573, 2012.

[17] R. Servadei and E. Valdinoci, "Mountain Pass solutions for nonlocal elliptic operators," Journal of Mathematical Analysis and Applications, vol. 389, no. 2, pp. 887-898, 2012.

[18] S. Palatucci, G. Dipierro, and E. Valdinoci, "Existence and symmetry results for a Schrödinger type problem involving the fractional Laplacian," Le Matematiche, vol. 68, no. 1, pp. 201-216, 2013.

[19] D. G. Costa, "On a class of elliptic systems in $\mathbb{R}^{N}$," Electronic Journal of Differential Equations, vol. 1994, no. 7, pp. 1-14, 1994.

[20] K. C. Chang, Methods in Nonlinear Analysis, Springer, Berlin, Germany, 2005. 


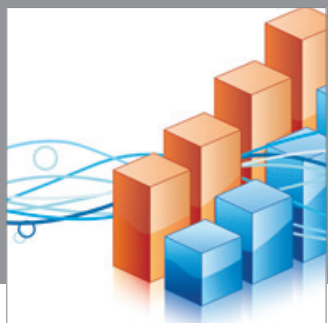

Advances in

Operations Research

mansans

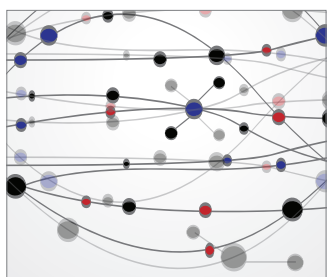

The Scientific World Journal
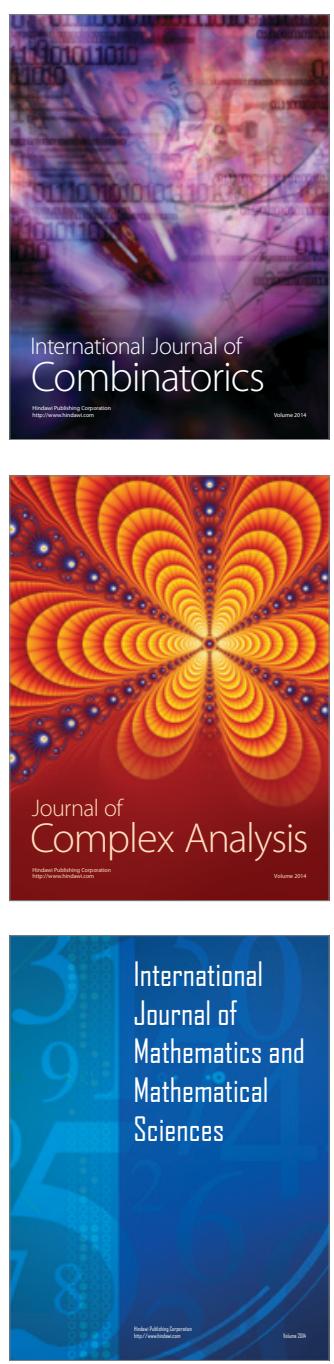
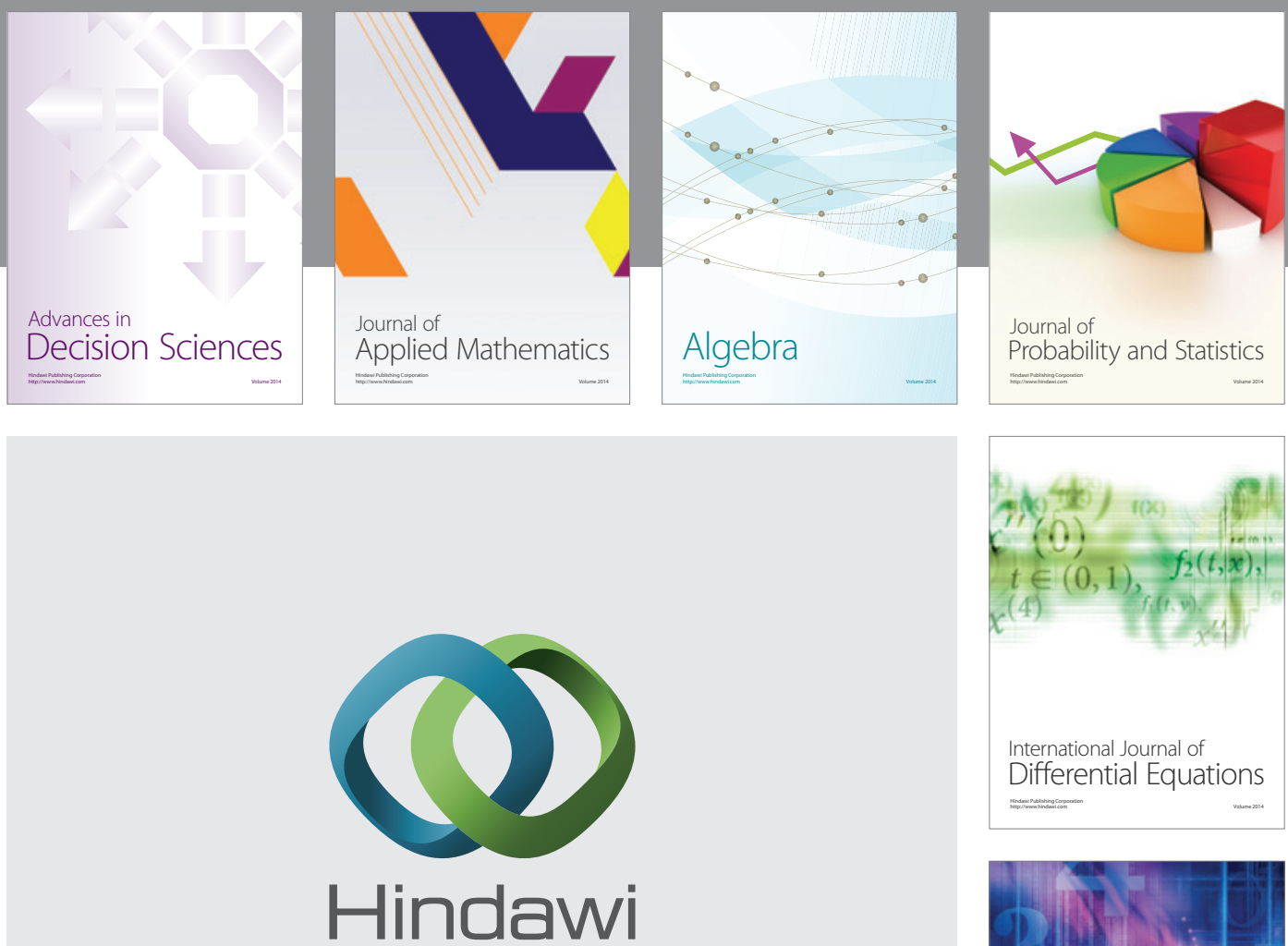

Submit your manuscripts at http://www.hindawi.com
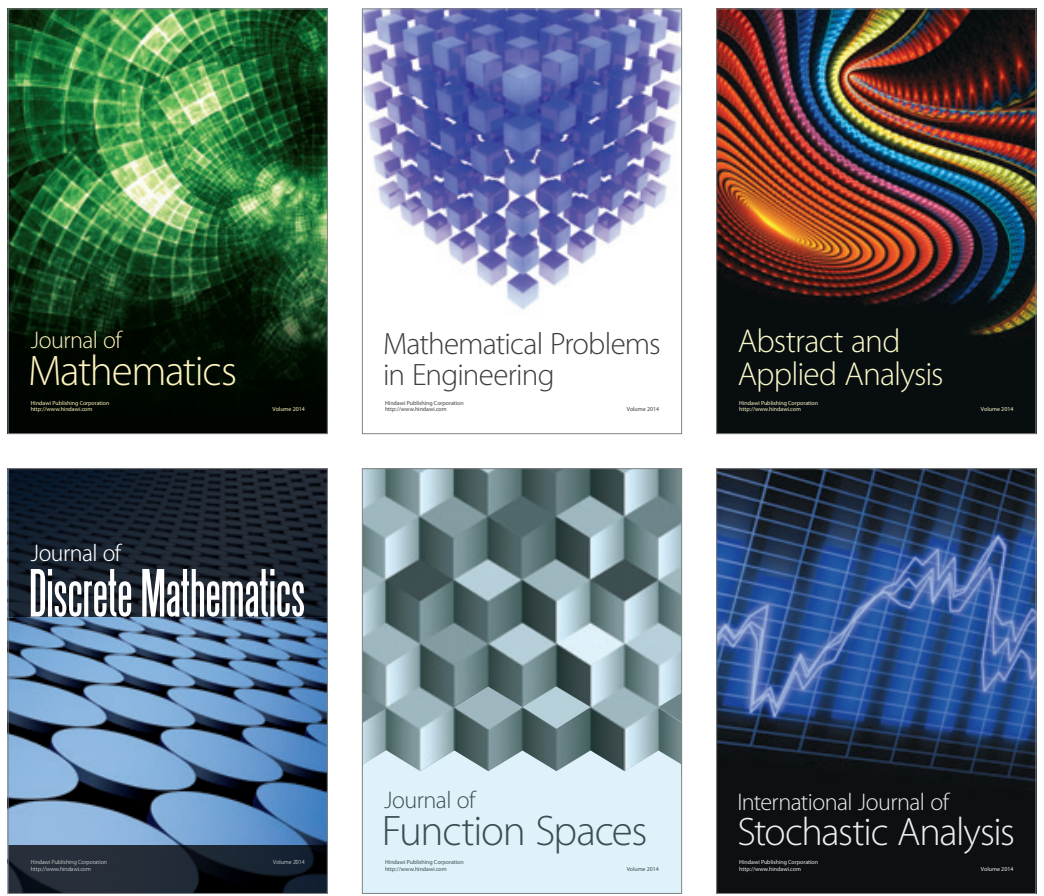

Journal of

Function Spaces

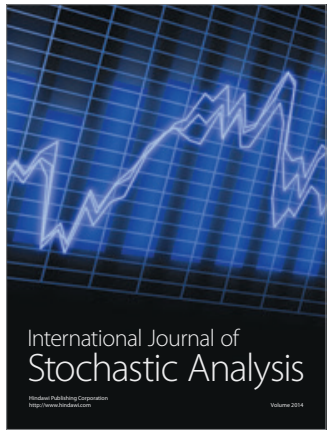

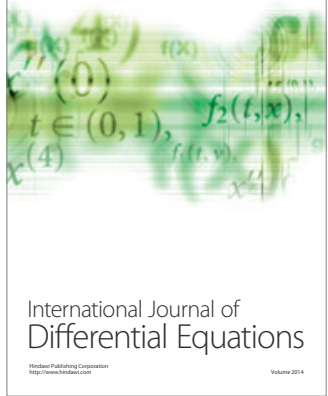
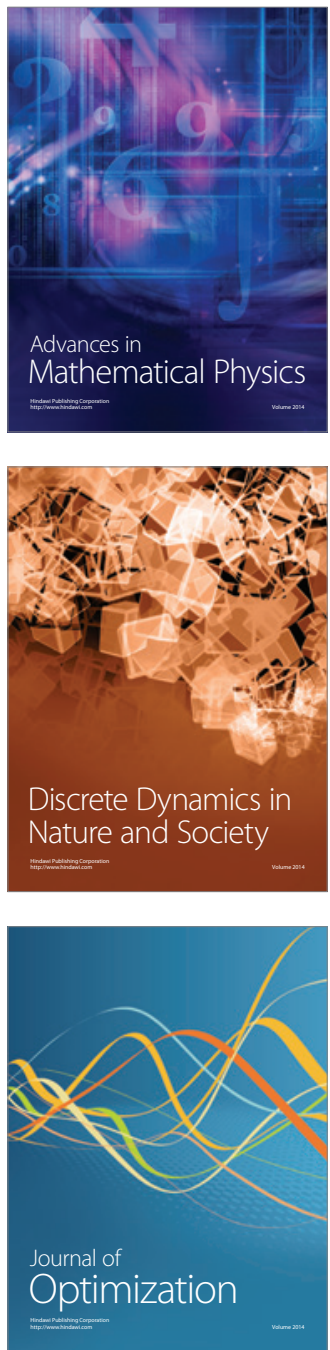